\title{
Group Consensus of Multi-agent Networks With Multiple Time Delays
}

\author{
Lianghao $\mathrm{Ji}^{1, *}$, Xinyue Zhao ${ }^{1}$, Qun Liu ${ }^{1}$ and Yong Wang ${ }^{2}$ \\ ${ }^{1}$ Chongqing Key Laboratory of Computational Intelligence, Chongqing University of Posts and Telecommunications, Chongqing \\ 400065, P. R. China \\ ${ }^{2}$ School of Computer Science and Technology, Chongqing University of Posts and Telecommunications, Chongqing 400065, P. \\ R. China
}

\begin{abstract}
This paper investigates the group consensus problem of first-order multi-agent networks with multiple time delays. By applying the theory of frequency-domain, we aim to propose some algebraic criteria such that the multi-agent networks can reach group consensus. From the results, it can be shown that group consensus of networks is determined by owning input time delays and connection strengths between agents, independent of communication delays. However, the existence of communication delays will affect the convergence rate of multi-agent networks. Finally, several numerical simulated examples are given to show the validity and correctness of our theoretical results.
\end{abstract}

Keywords-group consensus; multi-agent networks; time delays; complex network

\section{INTRODUCTION}

Partly due to broad applications of multi-agent networks in many areas such as congestion control[1] and flocking [2], the cooperative control has attracted many researchers' interest. As a fundamental branch of cooperative control, the consensus issues have become hot spots. Recently, many results about consensus problems have been established. Specific contents can be found in survey papers [3-4] and references therein, etc.

In cooperative control, in order to ensure target tasks to be completed harmoniously, it requires that states of all agents keep consistent with time. However, with the changes of environments, situations or even time, the consensus states are different. Generally, it can be described by group consensus problem. Up to date, group consensus of multi-agent networks has achieved some progress. Yu et al. [5,6] addressed group consensus with undirected or strongly connected and balanced graphs on the basis of the matrix theory. Furthermore, Yu et al. by the idea of double-tree-form transformation, extended group consensus problems with communication delays and switching topologies [7]. For special topologies with the same and different self-dynamic networks, the group consensus problems were investigated in [8]. Moreover, in order to reduce the cost of network control, pinning control strategies have been introduced into multi-agent networks. Particularly, the analysis of pinning group consensus started in [9-10] and references therein, etc. Additionally, under the strongly connected and balanced graph, Wang et al. [11] took group consensus with communication delays into consideration. Hu et al.[12] gave an overview of average-group consensus problems on networks with undirected topologies . Ji et al. provided group consensus with connected undirected and connected bipartite graphs respectively in [13]. In the connected bipartite graph, the reference [14] addressed group consensus of first-order networks with and without delays respectively by a control protocol. Moreover, Du et al. [15] extended the conclusions proposed in [14].

As we know, due to the communication link, equipment, etc., there exist two different kinds of delays in networks, that is communication delays and input delays. In fact, these two delays objectively exist and are different from each other. Thus the research towards group consensus of networks with multiple delays become more realistic. Comparing with existing investigations, there exist two main shortcomings: First, the effect of multi-agent networks with the two kinds of delays are considered insufficiently. Some related researches only involve communication delays, or only analyze the same communication and input delays [5-7,13-15]. Second, most of surveys only focus on networks with special topologies, such as undirected, strongly connected as well as strongly connected and balanced graphs, etc. [5-6,12-15]. Inspired by related researches, more general topologies are investigated. We address the group consensus issue with multiple delays on firstorder networks. Thereafter, algebraic criteria are derived that ensure group consensus to be achieved. Eventually, related results for similar issues are viewed as special cases of this paper, or analysis criteria are relatively less conservative.

The rest of the paper is organized as follows. In Section 2, relevant preliminaries on graph theory and model formulation are summarized. The problem of group consensus of networks with multiple time delays is discussed in Section 3. By a plurality of numerical experiments, the validity and accuracy of conclusions is verified in Section 4. Finally, concluding remarks and future trends are stated.

\section{PRELIMINARIES}

In multi-agent networks, the topology can be described by a directed graph. The node set is defined by $V=\left\{v_{1}, v_{2}, \ldots, v_{N}\right\}$. Denote the node indexes belong to a finite index set by $\aleph=\{1,2, \ldots, \mathrm{N}\}$ and the edges set is $E \subseteq V \times V$. The neighbor set of $v_{i}$ is $N_{i}=\left\{v_{j} \in V:\left(v_{i}, v_{j}\right) \in E\right\}$. Moreover, $A=\left\{a_{i j}\right\} \in \mathfrak{R}^{\mathrm{N} \times \mathrm{N}}$ is the weighted adjacency matrix. When 
$v_{j} \in N_{i}, a_{i j}>0$. For simplicity, we assume $\forall i \in \aleph, a_{i i}=0$. Let $D=\operatorname{diag}\left\{d_{i}, i \in \aleph\right\}$ be the degree matrix of $G, L=D-A$ is the Laplacian matrix of $G$.

For first-order networks, the dynamic is listed as (1):

$$
x_{i}(t)=u_{i}(t)
$$

where $x_{i}(t), u_{i}(t) \in \Re^{n}$ denotes the position, control input of the agent $i$. Without loss of generality, we assume $n=1$, i.e., we just only consider $x_{i}(t), u_{i}(t) \in \mathfrak{R}$. When $n>1$, by the Kronecker algorithm, it can be easily generalized.

Usually, for convenience, we restate some related lemma and definitions as follows.

Definition 1 The first-order multi-agent networks (1) is said to realize consensus asymptotically, if for any $i, j \in \aleph$, it follows that $\lim _{t \rightarrow \infty}=\left\|x_{i}(t)-x_{j}(t)\right\|=0$.

Definition $2^{[16]}$ For $G=(V, E, A)$, if $V$ is divided into two disjoint subgroups $\left\{V_{1}, V_{2}\right\}$, and the two vertices $v_{i}$ and $v_{j}$, associated with each edge $\left(v_{i}, v_{j}\right)$ respectively, belongs to two different sets of vertices, we call $G$ the bipartite graph.

Definition 3 If there exists a path in $G$ from $v_{i}$ to $v_{j}$, then $v_{j}$ is said to be reachable from $v_{i}$. If a node is reachable from every other node in $G$, then it is treated as a globally reachable node.

Lemma $\mathbf{1}^{[14]}$ If the topology of $G$ is a connected bipartite graph, the rank of $D+A$ is $n-1$.

Lemma ${ }^{[17]}$ If the graph $G$ exists a globally reachable node, its Laplacian matrix will have a simple eigenvalue 0.

Lemma $3^{[18]}$ For $\forall \gamma \in[0,1)$, when $\omega \in \mathfrak{R}$, convex hull $\gamma \operatorname{Co}\left(0 \mathrm{U}\left\{E_{i}(j \omega), i \in \aleph\right\}\right)$ does not contain $(-1, j 0)$, where $E_{i}(j \omega)=\frac{\pi}{2 T} \times \frac{e^{-j \omega T}}{j \omega}$ and $T$ denotes the system delay.

Lemma $4^{[19]}$ For $\omega \in \mathfrak{R}$, the set $U G_{i}$ is included in the convex hull $\gamma \operatorname{Co}\left(0 \mathrm{U}\left\{E_{i}(j \omega), i \in \mathfrak{\aleph}\right\}\right)$.

\section{GROUP CONSENSUS OF MULTI-AGENT NETWORKS WITH MULTIPLE DELAYS}

A. Group Consensus of Delayed Multi-agent Networks with the Connected Bipartite Topologies

For the network with the connected bipartite topology, ref. [15] by designing protocol (2), derived consistent states of convergence eventually. At the same time, it also discussed the maximum allowed delay such that the system (1) under protocol (3) can achieve group consensus.

$$
u_{i}(t)=-\sum_{v_{j} \in N_{i}} a_{i j}\left(x_{j}(t)+x_{i}(t)\right), i \in \aleph
$$

$$
u_{i}(t)=-\sum_{v_{j} \in N_{i}} a_{i j}\left(x_{j}(t-\tau)+x_{i}(t-\tau)\right), i \in \aleph
$$

where $\tau$ denotes the system delay.

By protocol (3), references [14-16] only discussed a special case of the same communication and input delays. Reference [15] did not give the conditions, where networks reached group consensus in the existence of time delays. In general, the two different kinds of delays exist objectively, so we derive some algebraic criteria. These algebraic criteria can guarantee group convergence of system (1) with protocol (4).

$$
u_{i}(t)=-\sum_{v_{j} \in N_{i}} a_{i j}\left(x_{j}\left(t-T_{i j}\right)+x_{i}\left(t-T_{i}\right)\right), i \in \aleph
$$

where $T_{i j}$ denotes communication delay and $T_{i}$ indicates input delay. With algorithm (4), the closed-loop form of (1) is

$$
x_{i}(t)=-\sum_{v_{j} \in N_{i}} a_{i j}\left(x_{j}\left(t-T_{i j}\right)+x_{i}\left(t-T_{i}\right)\right), i \in \aleph,
$$

Theorem 1 Assume the system (5) of $N$ agents with undirected bipartite topology. For $\forall i \in \aleph$, if $\max \left\{d_{i} T_{i}\right\}<\pi / 4$, system (5) can reach group consensus asymptotically .

Proof: By taking the Laplace transformation of (5), we obtain $\operatorname{det}\left(s I+D e^{-s T_{i j}}+A e^{-s T_{i}}\right)=0 \quad$.For simplicity, we define $F(s)=\operatorname{det}\left(s I+D e^{-s T_{i j}}+A e^{-s T_{i}}\right)$. By the general Nyquist stability criterion, the following two cases are discussed respectively:

1) When $s=0, F(s)=\operatorname{det}(D+A)$, based on Theorem 1 , we can derive that $F(s)$ has a simple zero at $s=0$.

2) When $s \neq 0$, let $P(s)=\frac{F(s)}{s}, G(s)=\frac{D e^{-s T_{i j}}+A e^{-s T_{i}}}{s}$. The discussion about the zeros of $F(s)$ equals to the zeros of $P(s)$. So if all zeros of $P(s)$ being on the open left complex plane, system (5) will have group consensus.

Let $s=j \omega$. From the Greshgorin disk theorem, the equivalent of $G(j \omega)$ satisfies 


$$
\begin{gathered}
\lambda(G(j \omega)) \in \cup_{i \in \aleph}^{\cup} G_{i}, \\
G_{i}=\left\{\zeta: \zeta \in C,\left|\zeta-\sum_{v_{j} \in N_{i}} a_{i j} \frac{e^{-j \omega T_{i}}}{j \omega}\right| \leq\left|\sum_{v_{j} \in N_{i}} a_{i j} \frac{e^{-j \omega T_{i}}}{j \omega}\right|\right\},
\end{gathered}
$$

where $C$ denotes complex field.

On the basis of (7), the center of the disk $G_{i}$ is $G_{i 0}(j \omega)=\sum_{v_{j} \in N_{i}} a_{i j} \frac{e^{-j \omega T_{i}}}{j \omega}$. Then intersection point is defined by $W_{i}$, which is made by the boundary of the disk and the origin point of the complex plane $O$. We can see that the track of point is $W_{i}(j \omega)=2 \sum_{v_{j} \in N_{i}} a_{i j} \frac{e^{-j \omega T_{i}}}{j \omega}$. From Lemma 3, noting that $W_{i}(j \omega)=\gamma_{i} \times E_{i}(j \omega)$, we know that for any given $\gamma_{i}<1$, it is easy to know that $\sum_{v_{j} \in N_{i}} a_{i j} T_{i}<\pi / 4$.

Now letting $\gamma=\max \left\{\gamma_{i}, i \in \aleph\right\}$, obviously, when $\gamma<1$, for any $i \in \mathfrak{N}$, it is easy to obtain that the next equation holds $\gamma \operatorname{Co}\left(0 \cup\left\{E_{i}(j \omega)\right\}\right) \supseteq \gamma_{i}\left(0 \cup\left\{E_{i}(j \omega)\right\}\right)=\operatorname{Co}\left(0 \cup\left\{W_{i}(j \omega)\right\}\right)$. In the view of Lemma 3, since $(-1, j 0) \notin \gamma \operatorname{Co}\left(0 \mathrm{U}\left\{E_{i}(j \omega), i \in \aleph\right\}\right)$, we also can conclude that $(-1, j 0) \notin \underset{i \in \mathbb{N}}{U} G_{i}$. Noting that $\operatorname{Co}\left(0 \cup\left\{W_{i}(j \omega), i \in \aleph\right\}\right) \supseteq \mathrm{U}_{i \in \mathbb{N}} G_{i}$, according to Lemma 4, we have $(-1, j 0) \notin \underset{i \in \mathbb{N}}{\mathrm{U}} G_{i}$. That is to say $\lambda(G(j w))$ does not contain $(-1, j 0)$. Therefore, we conclude that if the general Nyquist stability criterion is applied, all zeros of $P(s)$ have negative real parts. The proof of Theorem 1 is completed.

Remark 1 The allowable upper bounds of delays are proposed analytically, which can guarantee group consensus in [14]. In the sharp contrast to Lemma 1, the conclusion about the bound of delays is too broad. The following compared results of experiments verify the conclusion. Meanwhile, from the conclusion of Theorem 1, it shows that group convergence is subject to the input delays and adjacent weights, and is independent of communication delays.

Corollary 1 Supposed the system (5) of $N$ agents with directed bipartite topology, for $\forall i \in \aleph$, if $\max \left\{d_{i} T_{i}\right\}<\pi / 4$ is satisfied, system (5) can reach group consensus asymptotically.

The process of the proof is similar with Theorem 1 , we omit it due to the limitation of space.

\section{B. Group Consensus of Delay Systems with the Topology Owning a Globally Reachable Node}

Suppose the network consists of $n+m$ agents, and $L_{1}=\{1,2, \ldots, n\}, L_{2}=\{n+1, n+2, \ldots, n+m\}$. Based on the following two assumptions of in-degree balance , considering the following protocol (8) with multiple time delays,

a) $\sum_{j=n+1}^{n+m} a_{i j}=0, \forall i \in L_{1}$;b) $\sum_{j=1}^{n} a_{i j}=0, \forall i \in L_{2}$.

$u_{i}(t)=\left\{\begin{array}{l}\sum_{V_{j} \in N_{1 i}} a_{i j}\left(x_{j}\left(t-T_{i j}\right)-x_{i}\left(t-T_{i}\right)\right)+\sum_{V_{j} \in N_{2 i}} a_{i j} x_{j}\left(t-T_{i j}\right) \\ \sum_{V_{j} \in N_{2 i}} a_{i j}\left(x_{j}\left(t-T_{i j}\right)-x_{i}\left(t-T_{i}\right)\right)+\sum_{V_{j} \in N_{1 i}} a_{i j} x_{j}\left(t-T_{i j}\right)\end{array}\right.$

In (8), for $\forall i, j \in L_{1}, a_{i j} \geq 0 \quad ; \quad \forall i, j \in L_{2}, a_{i j} \geq 0 \quad$; $\forall i, j \in \phi=\left\{(i, j): i \in L_{1}, j \in L_{2}\right\} \bigcup\left\{(i, j): j \in L_{1}, i \in L_{2}\right\}, a_{i j} \in \mathfrak{R}$

With (8), the closed-loop form of (1) is

$$
x(t)=\left\{\begin{array}{l}
\sum_{V_{j} \in N_{1 i}} a_{i j}\left(x_{j}\left(t-T_{i j}\right)-x_{i}\left(t-T_{i}\right)\right)+\sum_{V_{j} \in N_{2 i}} a_{i j} x_{j}\left(t-T_{i j}\right) \\
\sum_{V_{j} \in N_{2 i}} a_{i j}\left(x_{j}\left(t-T_{i j}\right)-x_{i}\left(t-T_{i}\right)\right)+\sum_{V_{j} \in N_{1 i}} a_{i j} x_{j}\left(t-T_{i j}\right)
\end{array}\right.
$$

Theorem 2 Under assumptions of in-degree balance, we consider system (9) of $n+m(n, m>1)$ agents is a digraph which owns a globally reachable node. Then the network will achieve group consensus asymptotically if and only if $\max \left\{d_{i} T_{i}\right\}<\pi / 4, i=1,2, \ldots, n+m$, where $d_{i}=\sum_{k=1, k \neq i}^{m+n} a_{i k}$.

The proof progress of Theorem 2 is very similar to Theorem 1 and is omitted from this note due to the limitation of space.

\section{SimULATION EXAMPLES}

According to Theorem 1 and Theorem 2 respectively, some simulation examples are given to verify the effectiveness and the correctness of the criteria established above.

\section{A. Experiment $I$}

We consider system (5) with the topology and coupling weights between agents described in Figure I. Let $v_{1}, v_{2}$ in a group and $v_{3}, v_{4}, v_{5}$ in another group. The initial states of agents are $x(0)=[1.0,2.0,5.0,4.0,3.0]^{T}$. Input delays are $T_{1}=T_{2}=0.1 s, T_{3}=T_{4}=0.2 s, T_{5}=0.1 s$. In Figure II, it shows that the network can achieve group consensus. Comparing the network curves with different delays, it is obvious that the existence of communication delays can impact the convergence speeds of networks. 


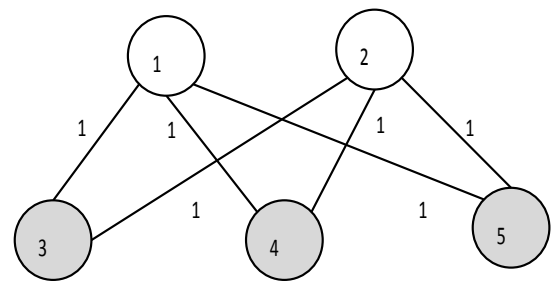

FIGURE I. TOPOLOGY OF SYSTEM (5)

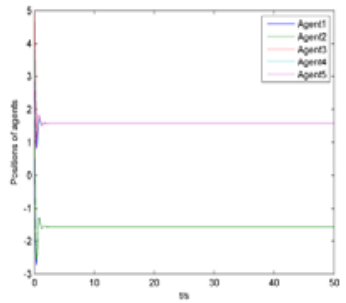

a. $T_{i j}=0.1 \mathrm{~s}$.

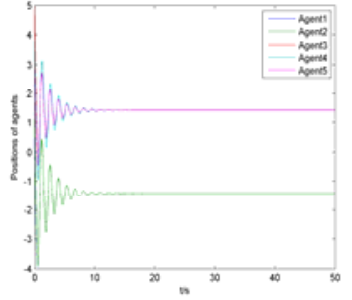

b. $T_{i j}=0.4 \mathrm{~s}$.
FIGURE II. TRAJECTORIES OF SYSTEM (5)

From Figure I, the degrees of $v_{1}$ and $v_{2}$ are 3. By Theorem 1 , in order to reach the group consensus of networks, the allowed input delays of $v_{1}$ and $v_{2}$ should hold $T_{i}<\pi / 12=0.26 s, i=1,2$. Based on the above experiments, we conduct experiments on the following situations. The state trajectories of system (5) are plotted in FigureIII.

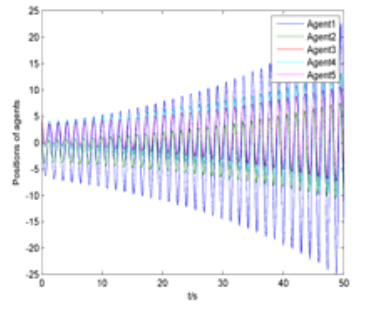

a. $T_{1}=0.26 \mathrm{~s}$.

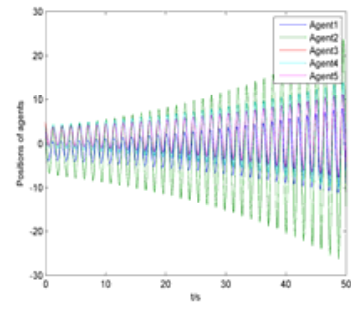

b. $T_{2}=0.26 \mathrm{~s}$.
FIGURE III. STATE TRAJECTORIES OF SYSTEM (5)

From FigureIII, it illustrates that system (5) will not reach group consensus. Compared with the result in [15], it is clear that the upper bound of the time delay we derived is more accurate.

\section{B. Experiment II}

We consider the topology of the network (11) with 5 nodes plotted in Figure IV. The initial states of agents are $x(0)=[2.0,3.0,5.0,7.0,6.0]^{T}$. Let $T_{i j}=0.4 s$, and input delays of each node are $0.6 \mathrm{~s}, 0.7 \mathrm{~s}, 0.3 \mathrm{~s}, 0.4 \mathrm{~s}$ and $0.1 \mathrm{~s}$, respectively. The state trajectories of (12) are shown in Figure V (a). The group consensus is achieved. By the condition of Theorem 2, we can learn that $d_{1}=1$ and the input delay satisfies $T_{1}<\pi / 4=0.785 \mathrm{~s}$. If set $T_{1}=0.79 \mathrm{~s}$, and the delays of other nodes keeping unchanged, from Figure V (b), we learn that the group consensus is fail to be realized. The correct and effective of Theorem 2 is verified.

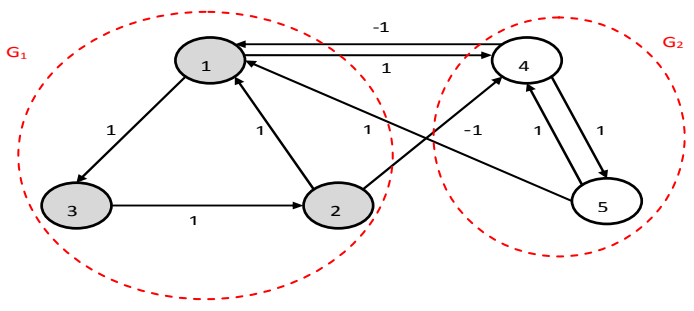

FIGURE IV. INTERCONNECTION GRAPH OF SYSTEM (11)

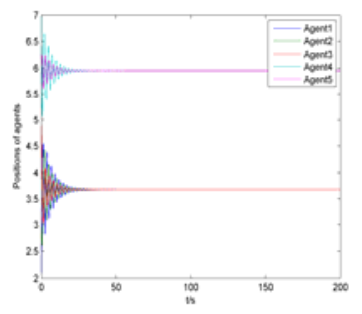

a. $T_{1}=0.6 \mathrm{~s}$

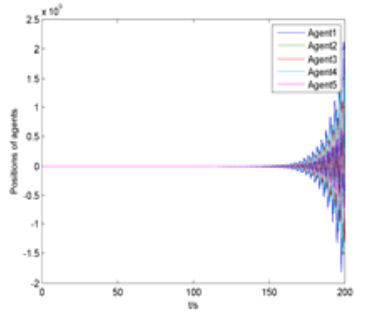

b. $T_{1}=0.79 \mathrm{~s}$.
FIGURE V. DYNAMIC BEHAVIORS OF SYSTEM (11)

\section{CONCLUSION}

For first-order networks, this technical survey is aimed at exploring the issue of group consensus of multi-agent networks with diverse communication and input delays. By the theory of frequency-domain, some algebraic criteria of the group consensus are derived. It can be shown that the group consensus of networks is determined by owning input delays and connection strengths, independent of communication delays. However, the existence of communication delays will affect the convergence speed of networks. Due to various reasons, the topology of complex networks typically changes, thus our future work will investigate the group consensus issue for diverse delays under switching topologies.

\section{ACKNOWLEDGMENT}

This work was supported by the National Natural Science Foundation of China (Grant No. 61472464), the Natural Science Foundation Project of Chongqing Science and Technology Commission (Grant Nos. cstc2014jcyjA40047, cstc2013jcyjA40017) and the Scientific and Technological Research Program of Chongqing Municipal Education Commission (Grant No. KJ1400403). The authors also would like to thank anonymous reviewers.

\section{REFERENCES}

[1] P. Yang, R. Freeman, G. Gordon, K. Lynch, S. Srinivasa, R. Sukthankar, "Decentralized estimation and control of graph connectivity for mobile sensor networks," Automatica, 2010, vol. 46, pp. 390-396.

[2] S. Charalambos, V. Vasos, P. Aristodemos, "Congestion control in wireless sensor networks through dynamic alternative path selection," Computer Networks, 2014, vol. 75, pp. 226-238. 
[3] T. Vicsek, A. Czirok, E. Ben-Jacob, O. Coben, I. Shochet, "Novel type of phase transition in a system of self-driven particles," Physical Review Letters, 1995, pp. 1226-1229.

[4] D. Lain, K. Jens, R. Nigel, A. Simon, "Effective leadership and decisionmaking in animal groups on the move,” Nature, 2005, pp. 513-516.

[5] J. Y. Yu, L. Wang, "Group consensus of multi-agent systems with undirected communication graph,” IEEE Xplore. China, pp. 105-110, August 2009 [Proceeding of the 7th Asian Control Conference. Hong Kong, China, 2009].

[6] J. Y. Yu, L. Wang, "Group consensus of multi-agent systems with directed information exchange," International Journal of Systems Science, vol. 43, pp. 334-348, August 2012.

[7] J. Y. Yu, L. Wang, "Group consensus in multi-agent systems with switching topologies and communication delays," System and Control Letters, vol. 59, pp. 340-348, June 2010.

[8] C. Tan, G. P. Liu, "Group consensus of networked multi-agent systems with directed topology,” Mllano, August 2011 [Preprints of the 18th IFAC World Congress, 2011].

[9] W. Wu, W. J. Zhou, T. P. Zhou, "Cluster Synchronization of Linearly Coupled Complex Networks Under Pinning Control," IEEE Transactions, vol. 56, pp. 829-839, April 2009.

[10] X. F. Liao, L. H. Ji, “On pinning group consensus for dynamical multiagent networks with general connected topology,” Neurocomputing, July 2014, pp. 262-267.

[11] M. H. Wang, k. Uchida, "Cluster Consensus of Multi-agent system with communication delay,” Control Automation and Systems, pp. 617-622, October 2013 [13th International conference on control, automation and systems, 2013].

[12] H. X. Hu, L. Yu, W. Zhang, H. Y. Song, "Group consensus in multiagent systems with hybrid protocol,” Journal of the Franklin Institute, vol. 350, pp. 575-597, April 2013.

[13] L. H. Ji, X. F. Liao, Q. Liu, "Group consensus analysis of multi-agent systems with delays,” Acta Phys Sin, vol. 61, 2012.

[14] Q. Wang, Y. Z. Wang, R. M. Yang, "Design and analysis of groupconsensus protocol for a class of multi-agent systems," Control and Decision, vol. 28, March 2013.

[15] X. Y. Du, Y. Z. Wang, Q. Wang, "Weighted group-consensus analysis of multi-agent systems with and without time-delay network," Control and Decision, pp. 6900-6905, July 2015.

[16] C. Godsil, G. Royle, Algebraic graph theory, New York: SpringerVerlag, 2001.

[17] W. Ren, R. W. Beard, “Consensus seeking in multiagent systems under dynamically changing interaction topologies," IEEE Transactions on Automatic Control, vol. 50, pp. 655-661, May 2005.

[18] Y. Tian, H. Yang, "Stability of distributed congestion control with diverse communication delays," Intelligent Control and Automation, vol. 2, pp. 1438-1442, June 2004 [the 5th World Congress on Intelligent Control and Automation, 2004].

[19] H. Y. Yang, S. W. Tian, S. Y. Zhang, "Consensus of multi-agent systems with heterogeneous delays and leader-following," Acta Electronica Sinica, vol. 39, pp. 872-876, 2011. 